\author{
A Tabu Search Heuristic for the Vehicle Routing Problem \\ Author(s): Michel Gendreau, Alain Hertz, Gilbert Laporte \\ Source: Management Science, Vol. 40, No. 10 (Oct., 1994), pp. 1276-1290 \\ Published by: INFORMS \\ Stable URL: http://www.jstor.org/stable/2661622 \\ Accessed: $11 / \overline{/ 03 / 2010 ~ 16: 39}$
}

Your use of the JSTOR archive indicates your acceptance of JSTOR's Terms and Conditions of Use, available at http://www.jstor.org/page/info/about/policies/terms.jsp. JSTOR's Terms and Conditions of Use provides, in part, that unless you have obtained prior permission, you may not download an entire issue of a journal or multiple copies of articles, and you may use content in the JSTOR archive only for your personal, non-commercial use.

Please contact the publisher regarding any further use of this work. Publisher contact information may be obtained at http://www.jstor.org/action/showPublisher?publisherCode=informs.

Each copy of any part of a JSTOR transmission must contain the same copyright notice that appears on the screen or printed page of such transmission.

JSTOR is a not-for-profit service that helps scholars, researchers, and students discover, use, and build upon a wide range of content in a trusted digital archive. We use information technology and tools to increase productivity and facilitate new forms of scholarship. For more information about JSTOR, please contact support@jstor.org. 


\title{
A Tabu Search Heuristic for the Vehicle Routing Problem
}

\author{
Michel Gendreau • Alain Hertz • Gilbert Laporte \\ Centre de recherche sur les transports Université de Montréal, C.P. 6128, succursale A, \\ Montréal, Québec, Canada H3C $3 J 7$ \\ Département de mathématiques, École Polytechnique Fédérale de Lausanne, \\ Ecublens, CH-1015 Lausanne, Switzerland
}

\begin{abstract}
$\mathrm{T}$ he purpose of this paper is to describe TABUROUTE, a new tabu search heuristic for the vehicle routing problem with capacity and route length restrictions. The algorithm considers a sequence of adjacent solutions obtained by repeatedly removing a vertex from its current route and reinserting it into another route. This is done by means of a generalized insertion procedure previously developed by the authors. During the course of the algorithm, infeasible solutions are allowed. Numerical tests on a set of benchmark problems indicate that tabu search outperforms the best existing heuristics, and TABUROUTE often produces the best known solutions.
\end{abstract}

(Vehicle Routing Problem; Tabu Search; Generalized Insertion)

\section{Introduction}

The purpose of this paper is to present TABUROUTE, a new heuristic for the following version of the Vehicle Routing Problem (VRP). Let $G=(V, A)$ be a directed graph where $V=\left\{v_{0}, v_{1}, \ldots, v_{n}\right\}$ is a vertex set, and $A=\left\{\left(v_{i}, v_{j}\right): i \neq j\right\}$ is an arc set. Vertex $v_{0}$ denotes a depot at which $m$ identical vehicles are based, and the remaining vertices of $V$ represent cities. The value of $m$ is either fixed at some constant, or bounded above by $\bar{m}$. With every $\operatorname{arc}\left(v_{i}, v_{j}\right)$ is associated a nonnegative distance $c_{i j}$. (For the sake of simplicity, the terms "distances," "travel times," and "travel costs" will be used interchangeably.) The VRP consists of designing a set of least cost vehicle routes in such a way that

(a) every route starts and ends at the depot;

(b) every city of $V \backslash\left\{v_{0}\right\}$ is visited exactly once by exactly one vehicle, and

(c) some side constraints are satisfied.

We consider the following side constraints:

(d) With every city is associated a nonnegative demand $q_{i}\left(q_{0}=0\right)$. The total demand of any vehicle route may not exceed the vehicle capacity $Q$. (e) Every city $v_{i}$ requires a service time $\delta_{i}\left(\delta_{0}=0\right)$. The total length of any route (travel plus service times) may not exceed a preset bound $L$.

In our version of the problem, vehicles bear no fixed cost, and their number is a decision variable.

The VRP lies at the heart of distribution management and has been extensively studied over the last three decades or so. (See the surveys by Christofides, Mingozzi, and Toth 1979, Bodin, Golden, Assad, and Ball 1983, Christofides 1985, Laporte and Nobert 1987, Golden and Assad 1988, and Laporte 1992). The VRP is a hard combinatorial problem, and to this day only relatively small VRP instances can be solved to optimality. Interesting exceptions are the problems solved to optimality by Fisher (1989), using minimum $k$-trees. We are mostly interested here in heuristic algorithms. Extending the scheme proposed by Christofides (1985), these algorithms can be broadly classified into four types: (1) Constructive algorithms (see, e.g., Clarke and Wright 1964, Mole and Jameson 1976, Desrochers and Verhoog 1989, Altinkemer and Gavish 1991); (2) Twophase algorithms (see, e.g., Gillett and Miller 1974, 
Christofides, Mingozzi, and Toth 1979, Fisher and Jaikumar 1981, Toth 1984); (3) Incomplete optimization algorithms (see, e.g., Christofides, Mingozzi, and Toth 1979); (4) Improvement methods (see, e.g., Stewart and Golden 1984; Harche and Raghavan 1991).

Metaheuristics such as simulated annealing and tabu search can be viewed as improvement methods. These are search schemes in which successive neighbors of a solution are examined, and the objective is allowed to deteriorate in order to avoid local minima. As a rule, these algorithms are designed to be open-ended and their running time, which can sometimes be quite large, is not a polynomial function of the size of the input data. Using an analogy with a material annealing process used in mechanics (Metropolis et al. 1953, Kirkpatrick, Gelatt, and Vecchi 1983), simulated annealing ensures that the probability of attaining a worse solution tends to zero as the number of iterations grows. Such a method was applied to the VRP by Osman (1991, 1993). Tabu search was proposed by Glover (1977) (see Glover 1989, 1990 and Glover, Taillard, and de Werra 1993 for recent overviews). Here, successive "neighbors" of a solution are examined and the best is selected. To prevent cycling, solutions that were recently examined are forbidden and inserted in a constantly updated tabu list. We are aware of a number of VRP algorithms based on this approach. One of the first attempts to apply tabu search to the VRP is due to Willard (1989). Here, the problem is first transformed into a TSP by replication of the depot, and the search is restricted to neighbor solutions that can be reached by means of 2-opt or 3-opt interchanges while satisfying the VRP constraints. In Pureza and França (1991), the search proceeds from one solution to the next by swapping vertices between two routes. Osman $(1991,1993)$ uses a combination of 2-opt moves, vertex reassignments to different routes, and vertex interchanges between routes. Another algorithm was developed by Semet and Taillard (1993) for the solution of a real-life VRP containing several features, and different from the version considered in this paper. Here the basic tabu move consists of moving a city from its current route into an alternative route. Finally, Taillard (1992) partitions the vertex set into clusters separately through vertex moves from one route to another. Clusters are updated throughout the algorithm. Note that in all these algorithms, a feasible solution is never allowed to become infeasible with respect to side constraints.

Our purpose is to describe a new tabu search procedure for the VRP. It differs from the implementations just described in several fundamental aspects. Our results show that the proposed algorithm is highly competitive on a set of benchmark problems. The remainder of this paper is organized as follows. The algorithm is presented in $\S 2$ and the computational results in $\S 3$. This is followed by the conclusion, in $\S 4$. We also provide, in an appendix, the best solutions obtained by our algorithm on the test problems.

\section{Algorithm}

This section contains a description of TABUROUTE followed by some comments. We use the following notation. A solution is a set $S$ of $m$ routes $R_{1}, \ldots, R_{m}$ where $m \in[1, \bar{m}], R_{r}=\left(v_{0}, v_{r_{1}}, v_{r_{2}}, \ldots, v_{0}\right)$, and each vertex $v_{i}(i \geq 1)$ belongs to exactly one route. These routes may be feasible or infeasible with respect to the capacity and length constraints. For convenience, we write $v_{i} \in R_{r}$ if $v_{i}$ is a component of $R_{r}$, and $\left(v_{i}, v_{j}\right) \in R_{r}$ if $v_{i}$ and $v_{j}$ are two consecutive vertices of $R_{r}$. With any feasible solution $S$, we associate the objective function

$$
F_{1}(S)=\sum_{r} \sum_{\left(v_{i}, v_{j}\right) \in R_{r}} c_{i j} .
$$

Also, with any solution $S$ (feasible or not), we associate the objective

$$
\begin{aligned}
F_{2}(S)= & F_{1}(S)+\alpha \sum_{r}\left[\left(\sum_{v_{i} \in R_{r}} q_{i}\right)-Q\right]^{+} \\
& +\beta \sum_{r}\left[\left(\sum_{\left(v_{i}, v_{j}\right) \in R_{r}} c_{i j}+\sum_{v_{i} \in R_{r}} \delta_{i}\right)-L\right]^{+},
\end{aligned}
$$

where $[x]^{+}=\max (0, x)$ and $\alpha, \beta$ are two positive parameters. If the solution is feasible, $F_{1}(S)$ and $F_{2}(S)$ coincide; otherwise, $F_{2}(S)$ incorporates two penalty terms for excess vehicle capacity and excess route duration. At any step of the algorithm, $F_{1}^{*}$ and $F_{2}^{*}$ denote respectively the lowest value of $F_{1}(S)$ and $F_{2}(S)$ so far encountered. Also, $S^{*}$ is the best known feasible solution and $\tilde{S}^{*}$, the best known solution (feasible or not).

We first describe procedure SEARCH $(P)$, central to TABUROUTE. This procedure attempts to improve upon a given solution $S$, using tabu search. It calls GENI 
and US, two heuristics developed by the authors for the TSP (Gendreau, Hertz, and Laporte 1992). The first, GENI, is a generalized insertion routine. It is less myopic but more powerful than standard insertion procedures in that a vertex may be inserted only into a route containing one of its closest neighbors, and every insertion is executed simultaneously with a local reoptimization of the current tour. US is a post-optimization procedure that successively removes and reinserts every vertex, using GENI. Again, US has produced highly satisfactory results on the TSP, better than Or-opt, for example. The combination of GENI and US yields a powerful twophase heuristic for the TSP. SEARCH is governed by a vector of parameters

$$
P=\left(W, q, p_{1}, p_{2}, \theta_{\min }, \theta_{\max }, g, h, n_{\max }\right)
$$

defined as follows:

$W$ : a nonempty subset of $V \backslash\left\{v_{0}\right\}$ containing vertices that are allowed to be moved from their current route;

$q$ : number of vertices of $W$ that are candidate for reinsertion into another route;

$p_{1}$ : the route in which vertex $v$ is reinserted must contain at least one of its $p_{1}$ nearest neighbors;

$p_{2}$ : neighborhood size used in GENI;

$\theta_{\min }, \theta_{\max }$ : bounds on the number of iterations for which a move is declared tabu;

$g$ : a scaling factor used to define an artificial objective function value;

$h$ : the frequency at which updates of $\alpha$ and $\beta$ are considered;

$n_{\max }$ : maximum number of iterations during which the last step of the procedure is allowed to run without any improvement in the objective function.

PROCEDURE SEARCH $(P)$

Step 0 (Initialization). Set the iteration count $t:=1$, no move is tabu.

Step 1 (Vertex selection). Consider solution $S$ and randomly select $q$ cities from $W$.

Step 2 (Evaluation of all candidate moves). Repeat the following procedure for all selected vertices $v$.

Consider all potential moves of $v$ from its current route $R_{r}$ into another route $R_{s}$ containing no city (if $m$ $<\bar{m}$ ), or at least one of the $p_{1}$ nearest neighbors of $v$. Repeat the following operations for all candidate moves:

(a) Remove $v$ from $R_{r}$ and compute its insertion cost into $R_{s}$, using the GENI algorithm with parameter $p_{2}$ and determine the corresponding $S^{\prime}$.

(b) If the move is tabu, it is disregarded unless $S^{\prime}$ is feasible and $F_{1}\left(S^{\prime}\right)<F_{1}^{*}$, or $S^{\prime}$ is infeasible and $F_{2}\left(S^{\prime}\right)$ $<F_{2}^{*}$.

(c) Otherwise, $S^{\prime}$ is assigned a value $F\left(S^{\prime}\right)$ equal to $F_{2}\left(S^{\prime}\right)$ if $F_{2}\left(S^{\prime}\right)<F_{2}(S)$, or to $F_{2}\left(S^{\prime}\right)+\Delta_{\max } \sqrt{m} g f_{v}$ otherwise, where $\Delta_{\max }$ is the largest observed absolute difference between the values of $F_{2}(S)$ obtained at two successive iterations, and $f_{v}$ is the number of times vertex $v$ has been moved, divided by $t$.

Step 3 (Identification of best move). The candidate move yielding the least value of $F$ and solution $\bar{S}$ is identified.

Step 4 (Next solution). The move identified in Step 3 is not necessarily implemented. It may indeed be advantageous to attempt to improve $S$ by applying to each individual route of $S$ the US post-optimization procedure described in Gendreau, Hertz, and Laporte (1992). Solution $S$ is set equal to $\bar{S}$, unless the following three conditions are satisfied: (a) $F_{2}(\bar{S})>F_{2}(S)$; (b) $S$ is feasible; (c) US has not been used at iteration $t-1$; in such a case $S$ is obtained by applying the US postoptimization process.

Step 5 (Update). If the US procedure has not been used in Step 4 and vertex $v$ has been moved from route $R_{r}$ to route $R_{s}(s \neq r)$, reinserting $v$ into $R_{r}$ is declared tabu until iteration $t+\theta$, where $\theta$ is an integer randomly selected in $\left[\theta_{\min }, \theta_{\max }\right]$. Set $t:=t+1$, update $F_{1}^{*}, F_{2}^{*}$, $S^{*}, \tilde{S}^{*}, \Delta_{\max }, m$ and $f_{v}$.

Step 6 (Penalty adjustment). If $t$ is a multiple of $h$, adjust $\alpha$ and $\beta$ as follows. Check whether all previous $h$ solutions were feasible with respect to vehicle capacity. If so, set $\alpha:=\alpha / 2$; if they were all infeasible, set $\alpha:=2 \alpha$. Similarly, if all previous $h$ solutions were feasible with respect to route length, set $\beta:=\beta / 2$; if they were all infeasible, set $\beta:=2 \beta$.

Step 7 (Termination check). If $F_{1}^{*}$ and $F_{2}^{*}$ have not decreased for the last $n_{\max }$ iterations, stop. Otherwise, go to step 1 .

The main algorithm can now be described. At first, several tentative initial solutions are generated, SEARCH is applied to each of them for a limited number of iterations, and the most promising solution is selected as a starting point for TABUROUTE. Procedure SEARCH is then called twice with different values $P_{1}$ 
and $P_{2}$ of the parameters. The first call usually brings the most significant improvement to the initial solution, while the second call intensifies the search locally by concentrating on specific subsets of cities of the best known feasible solution if any, or of the best known infeasible solution otherwise.

\section{ALGORITHM TABUROUTE}

Step 0 (Initialization). Set $\alpha:=\beta:=1$ and $F_{1}^{*}:=\infty$. If vertices are described by two-dimensional coordinates, relabel them according to the angle they make with the depot and a horizontal line.

Step 1 (First solution). Repeat the following operations $\lambda$ times, where $\lambda$ is an input parameter.

(a) Randomly select a city $v_{i}$.

(b) Using the vertex sequence

$$
\left(v_{0}, v_{i}, v_{i+1}, \ldots, v_{n}, v_{1}, \ldots, v_{i-1}\right),
$$

construct a tour on all vertices by means of the GENIUS heuristic for the TSP (Gendreau, Hertz, and Laporte 1992).

(c) Starting with $v_{0}$, create at most $\bar{m}$ vehicle routes by following the tour: the first vehicle contains all cities starting from the first city on the tour and up to, but excluding, the first city $v_{i}$ whose inclusion in the route would cause a violation of the capacity or maximal length constraint; this process is then repeated, starting from $v_{i}$, and until all cities have been included into routes (the solution is then feasible), or until $\bar{m}-1$ vehicles have been used, in which case all remaining cities are assigned to vehicle $\bar{m}$ (the solution may then be infeasible). Let $S$ be the solution (feasible or not) obtained through this process. Update $F_{1}^{*}, F_{2}^{*}, S^{*}$ and $\tilde{S}^{*}$.

(d) Call SEARCH $\left(P_{1}\right)$.

(e) If $F_{1}^{*}<\infty$, set $S:=S^{*}$; otherwise, set $S:=\tilde{S}^{*}$.

Step 2 (Solution improvement). Call SEARCH $\left(P_{2}\right)$. If $F_{1}^{*}<\infty$, set $S:=S^{*}$; otherwise, set $S:=\tilde{S}^{*}$.

Step 3 (Intensification). Call SEARCH $\left(P_{3}\right)$. If $F_{1}^{*}<\infty$, $S^{*}$ is the best known feasible solution; otherwise, no feasible solution has been found.

Stop.

We now comment on the choice of parameters used in SEARCH and TABUROUTE, and on a number of algorithmic aspects. As far as parameters are concerned, we have selected them independently of the test problems, relying as much as possible on theoretical consid- erations and on the experience developed by other researchers in the field of tabu search. In a limited number of cases where no firm basis existed for choosing the parameters, we have selected reasonable a priori values, and sensitivity analyses were then conducted on all test problems.

Step 2c of SEARCH contains a diversification strategy. Following Glover (1989), vertices that have been moved frequently are penalized by adding to the objective function of the candidate solution a term proportional to the absolute frequency of movement of the vertex $v$ currently being moved. Taillard (1992) suggests using a constant equal to the product of three factors: (a) $\Delta_{\max }$, a factor equal to the absolute difference value between two successive values of the objective function, (b) the square root of the neighborhood size (shown later to be proportional to the number $m$ of routes), (c) a scaling factor $g$ equal to 0.01 in our implementation. As a rule, using too large a value of $g$ lessens the likelihood of obtaining a good solution. Too low a value does not produce the desired diversification effect because the algorithm does not move away from the current solution. Post-optimality tests show that the algorithm is quite insensitive to $g$ as long as it remains in the interval [0.005, 0.02].

The variable tabu list length $(\theta)$ used in Step 5 of SEARCH was also inspired from Taillard's work (1991). After extensive experiments on the application of tabu search to the quadratic assignment problem, this author concludes that the probability of obtaining a global optimum is increased in the case of a variable list length. Our implementation of random duration tabus differs from that proposed by Taillard since no tabu list is actually used. Instead, each move individually receives a random duration tabu tag denoted $\theta$ : this limits the amount of bookkeeping required and, as a result, the speed of the algorithm is increased. In the current implementation, we use $\theta_{\min }=5$ and $\theta_{\max }=10$, as suggested by Glover and Laguna (1993) for "simple dynamic tabu term rules."

The idea used in Step 6 of SEARCH of updating $\alpha$ and $\beta$ during the course of the algorithm could also be applied to other contexts where penalty terms are added to the objective function. All too often, choosing an appropriate coefficient value is difficult, and a wrong choice can have an adverse impact on the performance 
of the algorithm. Here penalty coefficients are doubled if the $h=10$ previous solutions were infeasible and halved if the $h=10$ previous solutions were feasible. With this rule, we quickly arrive at values of $\alpha$ and $\beta$ that produce a mix of feasible and infeasible solutions. We found the algorithm is not very sensitive to the value of $h$. Thus, solutions produced with $h=5$ or 20 are at most $1 \%$ worse than those obtained with $h=10$. In this type of algorithm, obtaining infeasible solutions is important since this helps moving out of local optima. Hertz (1992) uses this idea in the context of a course scheduling algorithm.

We now comment on algorithm TABUROUTE. The value currently used for $\lambda$, the number of tentative initial solutions, is equal to $[\sqrt{n} / 2]$. Post-optimality tests indicate that it pays to use a value of $\lambda$ greater than 1 and as large as $[\sqrt{n} / 2]$, because the algorithm is then less likely to start on the wrong track. Values of $\lambda$ larger than $[\sqrt{n} / 2]$ were also tested, but as a rule the extra computational effort required is not justified by the quality of the results.

The idea of using a tour construction heuristic, as in Step 1c of TABUROUTE, has already been used by a number of researchers (see, e.g., Beasley 1983 and Haimovich and Rinnooy Kan 1985). Our implementation is different in that we resort to the more powerful GENIUS algorithm to obtain an initial tour. When the number of available vehicles is unbounded (i.e., $\bar{m}=n$ ), the initial solution is always feasible. However, for smaller values of $\bar{m}$, feasibility at this stage is not guaranteed, because the problem of finding a feasible solution to the capacity constrained VRP is a bin packing problem and is therefore NP-complete (Garey and Johnson 1979). Comparisons were made with a simplified version of the algorithm using random starting solutions. More precisely, for each solution 50 routes were initialized with a randomly selected seed, and the remaining vertices were then arbitrarily inserted into the existing routes. Results show that the final solution values obtained using the procedure described in Step 1 of TABUROUTE are approximately $1 \%$ better than those obtained from randomly generated routes.

We now discuss the choice of parameters $W, q, p_{1}$, $p_{2}$, and $n_{\max }$ in the various calls to SEARCH $(P)$. Parameter $W$ defines the subset of cities that can be moved into different routes in procedure SEARCH. This parameter is always equal to $V \backslash\left\{v_{0}\right\}$, except in the intensification step of TABUROUTE (Step 3), where $W$ is defined as the $\lfloor|V| / 2\rfloor$ vertices $v$ with the largest $f_{v}$; these vertices have often been moved and are therefore likely to yield a solution improvement if moved. In Step 3 , the value of $q$ is equal to $|W|$. In other words, all vertices that are allowed to move are candidates for reinsertion. In $P_{1}$ and $P_{2}$, however, it would be prohibitive to consider so many reinsertions. Here, $q$ is chosen to ensure a sufficiently high probability of selecting at least one vertex from each route. This probability is $P(q, m)=S(q, m) m ! / m^{q}$ (assuming the number of cities in each route is sufficiently large), where $S(q, m)$ is a Stirling number of the second kind (Riordan 1958). The most appropriate value of $q$ depends on $m$; as long as $m \leq 30$, taking $q=5 m$ ensures that $P(q, m) \geq 0.9$. Parameter $p_{2}$ corresponds to the neighborhood size in GENI. Extensive tests performed by Gendreau, Hertz, and Laporte (1992) indicate that taking $p_{2}=5$ ensures that a near-optimal TSP solution will be found relatively quickly; this is the value used in $P_{1}, P_{2}$, and $P_{3}$. The algorithm is quite sensitive to the value of this parameter. Using $p_{2} \leq 4$ tends to produce low quality solutions; in contrast, when $p_{2} \geq 6$, running times become excessive.

Parameter $p_{1}$ is equal to $\max \left(k, p_{2}\right)$, where $k$ is the number of cities in the route containing the vertex $v$ currently being moved. This value of $p_{1}$ ensures that at least one potential move will relocate $v$ into a different route. Finally, the value of $n_{\max }$ is equal to $n$ in $P_{1}, P_{3}$, and to $50 \mathrm{n}$ in $P_{2}$, as the most important part of the search is executed in Step 2. The running time of the algorithm is linearly related to the value of this parameter in $P_{2}$. If $n_{\max }$ is too low, some good solutions will be missed. If it is too high, there is a risk that the algorithm will run a long time without improvement. Sensitivity analyses performed on all test problems suggest $50 n$ is a good compromise.

\section{Computational Results}

TABUROUTE was tested on the fourteen test problems described in Christofides, Mingozzi, and Toth (1979). These problems contain between 50 and 199 cities in 
addition to the depot. Problems 1-5 and 11-12 have capacity restrictions only. Problems 6-10 are the same as $1-5$, except that they have a route length constraint as well; problems 13-14 are also the same as 11-12, with a route length constraint. In problems 1-10, cities are randomly generated in the plane, while in problems 11-14, they appear in clusters. All computations were executed with distances rounded up or down after four decimals. The final solutions were evaluated with real distances, and the objective value was then rounded up or down after two decimals.

Comparisons were made between TABUROUTE and other heuristic algorithms for which computational results have already been published for the same problems:

CW: the Clarke and Wright (1964) savings algorithm;

MJ: $\quad$ the Mole and Jameson (1976) generalized savings algorithm;

AG: $\quad$ the Altinkemer and Gavish (1991) PSA-T algorithm;

DV: $\quad$ the Desrochers and Verhoog (1989) MBSA algorithm;

GM: the Gillett and Miller (1974) SWEEP algorithm;

CMT1: the Christofides, Mingozzi, and Toth (1979) two-phase algorithm;

FJ: $\quad$ the Fisher and Jaikumar (1981) two-phase algorithm;

CMT2: the Christofides, Mingozzi, and Toth (1979) incomplete tree search algorithm;

OSA: Osman's (1993) simulated annealing algorithm;

PF: the Pureza and França (1991) tabu search algorithm;

OTS: Osman's (1993) tabu search algorithm;

T: $\quad$ Taillard's (1992) tabu search algorithm.

Solution values for these algorithms are reported in Table 1. These values are extracted from the respective references except for $\mathrm{CW}, \mathrm{MJ}$, and GM, which are taken from Christofides, Mingozzi, and Toth (1979).

We report two sets of figures for TABUROUTE. The "standard" column contains results for a single pass of TABUROUTE, using the parameters described in $\S 2$. However, in the course of performing the various sen- sitivity analyses, we did on occasions produce better solutions; the corresponding local optima are reported in column "best." Asterisks correspond to the best verifiable solutions obtained with real $c_{i j}$ s. The full solutions for the "best" column are reported in the appendix.

These results show that all "classical" algorithms (CW to CMT2 in Table 1) are clearly dominated by simulated annealing and tabu search, as far as solution values are concerned. TABUROUTE is highly competitive and generally produces the best known solutions. However, when analyzing results, care must be taken to make equitable comparisons. Thus, "TABUROUTE standard" executes a single pass with a priori parameters, while for other columns (e.g., AG, OSA, OTS, T, and "TABUROUTE best") the algorithm was run for several variants, and the best solution was selected. Similarly, parameters in some algorithms are undefined in the original article, and the rule for selecting seed points in FJ is not well specified. Another problem arises from the type of distances that were used. It must first be said that we did not generally possess the full solutions produced by the other algorithms, but only their value. This poses a number of difficulties. It is obvious that rounding or truncating must have occurred in the final solution value, on the individual route costs, or on the distances themselves since the reported optima are often integer while the original distances are real. As a result, the integer values reported in Table 1 may underestimate the true value to some extent. To our knowledge, only the columns OSA, OTS, T, and TABUROUTE correspond to verifiable solutions obtained with real $c_{i j} \mathrm{~s}$. The effect of rounding and truncating is best illustrated on problem 1 . When this problem is solved with real $c_{i j} \mathrm{~s}$, a feasible solution of cost 524.61 is obtained. Recently, Hadjiconstantinou and Christofides (1993) have proved this result is optimal. Using rounded costs, TABUROUTE produces a solution of value 521, again a proven optimum (Cornuejols and Harche 1993). The same value is given by Fisher (1989) without specification of the rounding convention employed, and by other authors who worked with rounded distances (Harche and Raghavan 1991, Noon, Mittenthal, and Pillai 1991). Using truncated costs, we obtain an objective value of 508 with TABUROUTE. Another difficulty arises in problems with very tight route length 


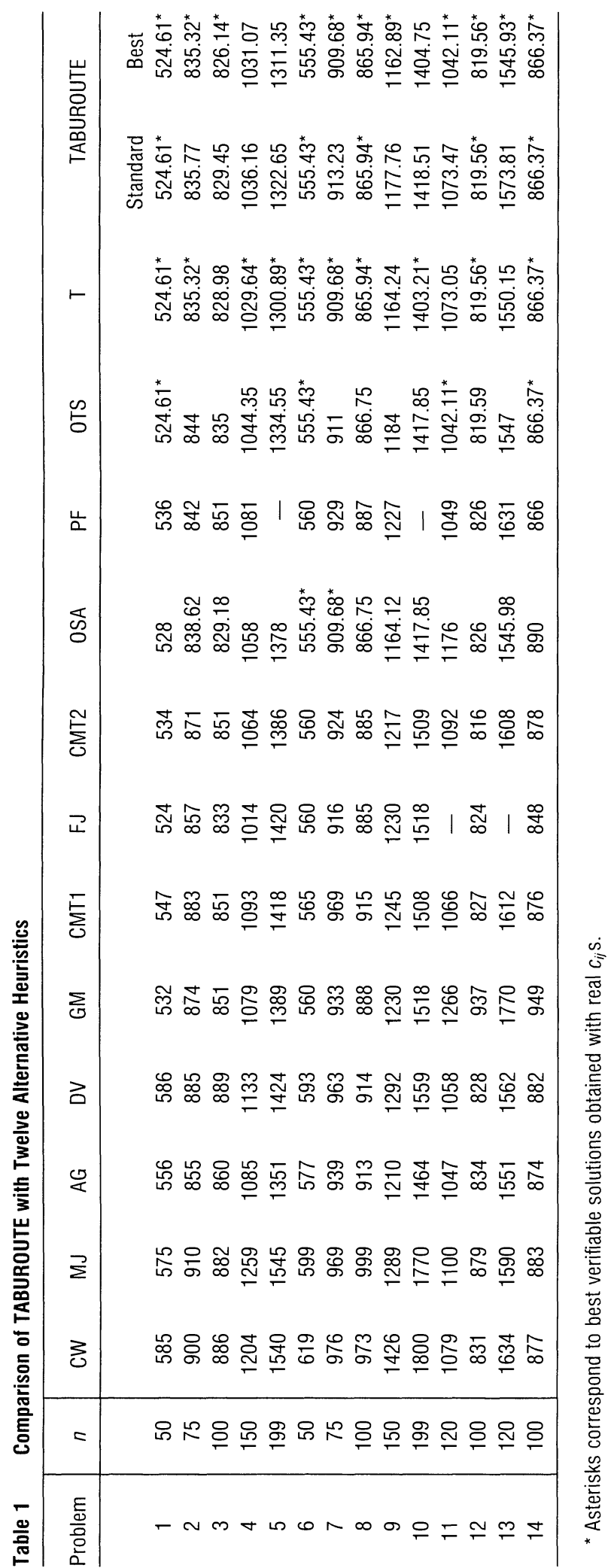

constraints, where some routes can be infeasible for TABUROUTE, but feasible when rounding or truncating occurs. We are aware of such cases where TABUROUTE would have achieved a much better value had we considered legal some routes with a length exceeding $L$ by less than one unit. This type of problem has already been reported by Mole (1983) in relation with a vehicle scheduling algorithm by Cheshire, Malleson, and Naccache (1982). We also compared TABUROUTE with algorithms known to have been tested with truncated distances (Toth 1984) or with rounded distances (Harche and Raghavan 1991, Noon, Mittenthal, and Pillai 1991) by using the same type of distance. In each case, TABUROUTE produced better or identical results on all 14 problems.

The methodological problems just raised make direct computation time comparisons difficult, particularly when an unspecified number of passes of the same algorithm were executed with different parameters, or when inordinate computing times were allowed. In addition, at least one algorithm ( $T$ ) uses parallel computing. By and large, metastrategies such as simulated annealing and tabu search require higher computation times than classical heuristics, but given the vast improvements in solution quality, we feel the extra computational effort is well justified. We report in Table 2 the computation times in minutes on a Silicon Graphics workstation, $36 \mathrm{MHz}$, 5.7Mflops, for the standard version of TABUROUTE. More specifically, we show the times required to compute the $\lambda$ initial solutions, to reach the best encountered solution, and to terminate the algorithm. These results show that the relationship between problem size and computation time is not monotonous, and the moment at which the best solution is identified is quite unpredictable. Thus, in problems 4 and 5 , it is encountered toward the end of the search process, while in problem 12, it is discovered at an early stage, during the initial trials.

\section{Conclusion}

We have described in this paper a new tabu search algorithm for the VRP. Results obtained on a series of benchmark problems indicate clearly that tabu search outperforms the best existing heuristics, and TABUROUTE often produces the best known solutions. By nature, tabu search is a metaheuristic that must be tai- 
Table 2 Computation Times for the Standard Version of TABUROUTE

\begin{tabular}{rrrrr}
\hline & \multicolumn{4}{c}{ Computation Times in Minutes } \\
\cline { 3 - 5 } & & $\begin{array}{c}\text { For the } \lambda \\
\text { Initial Trials }\end{array}$ & $\begin{array}{c}\text { To Obtain the } \\
\text { Best Solution }\end{array}$ & Total \\
\hline & & & & \\
\hline & & & & \\
2 & 50 & & 1.4 & 6.0 \\
3 & 75 & 2.6 & 39.2 & 53.8 \\
4 & 100 & 3.1 & 6.8 & 18.4 \\
5 & 150 & 7.4 & 54.5 & 58.8 \\
6 & 199 & 15.9 & 83.8 & 90.9 \\
7 & 50 & 1.1 & 7.8 & 13.5 \\
8 & 75 & 3.2 & 31.8 & 54.6 \\
9 & 100 & 3.9 & 5.9 & 25.6 \\
10 & 150 & 11.9 & 21.3 & 71.0 \\
11 & 199 & 21.4 & 44.1 & 99.8 \\
12 & 120 & 3.0 & 11.9 & 22.2 \\
13 & 100 & 3.5 & 1.7 & 16.0 \\
14 & 120 & 10.3 & 34.8 & 59.2 \\
\hline
\end{tabular}

lored to the shape of the particular problem at hand. We attribute a large part of the success of TABUROUTE to at least two main implementation devices. One is the fact that we allow infeasible solutions through penalty terms in the objective function, thus reducing the likelihood of local minima. A second important ingredient of our method is the use of GENI to execute the insertions. Not only does this help produce better tours, but as a result, the solution is periodically perturbed and thus the risk of being trapped in a local optimum is again reduced. Finally, one major advantage of the proposed algorithm lies in its flexibility. It can be executed from any starting solution (feasible or not); it can also be adapted to contexts where the number of vehicles is fixed or bounded, where vehicles have different characteristics, etc. Also, additional features can easily be handled, such as assigning particular cities to specific vehicles, using several depots, allowing for primary and secondary routes, and so on. ${ }^{1}$

${ }^{1}$ This work was partially supported by the Canadian Natural Sciences and Engineering Research Council under grants OGP0038816, OGP0039682, and OGP0105384. The second author also benefitted from an NSERC International Fellowship. Thanks are also due to the referees for their valuable comments.

\section{Appendix. Best Solutions Obtained with Taburoute (real distances)}

The "Time" column shows travel times only. To obtain total route durations, service times must be added, where applicable.

Problem 1

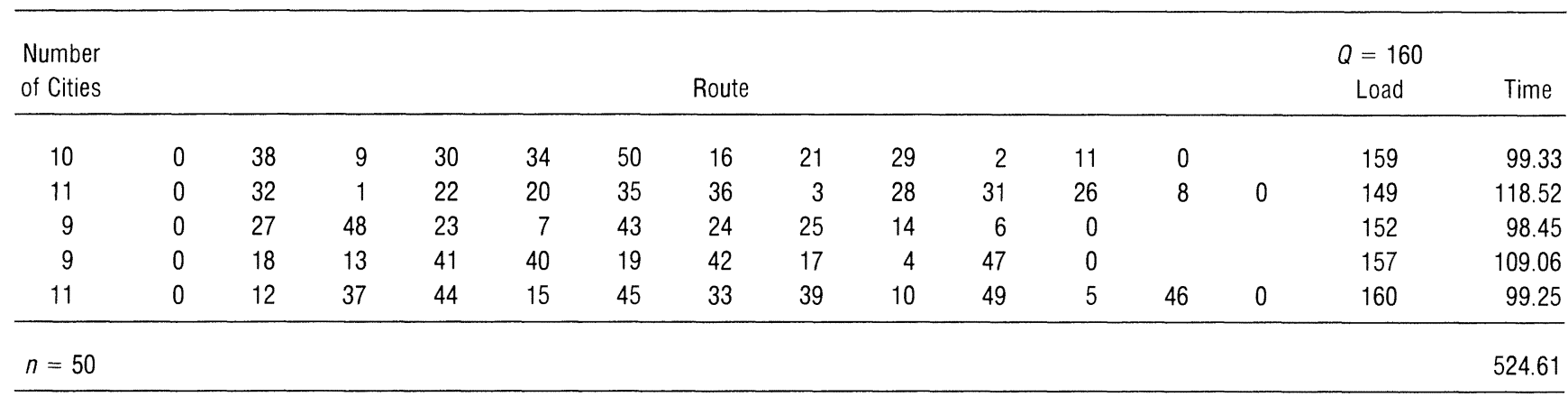


Problem 2

\begin{tabular}{|c|c|c|c|c|c|c|c|c|c|c|c|c|c|c|}
\hline 5 & 0 & 17 & 40 & 32 & 9 & 39 & 0 & & & & & & 126 & 57.01 \\
\hline 6 & 0 & 7 & 53 & 11 & 10 & 58 & 26 & 0 & & & & & 139 & 71.90 \\
\hline 6 & 0 & 38 & 65 & 66 & 59 & 14 & 35 & 0 & & & & & 135 & 93.03 \\
\hline 7 & 0 & 30 & 74 & 21 & 69 & 61 & 28 & 2 & 0 & & & & 138 & 88.71 \\
\hline 9 & 0 & 73 & 1 & 43 & 41 & 42 & 64 & 22 & 62 & 68 & 0 & & 136 & 94.63 \\
\hline 8 & 0 & 6 & 33 & 63 & 23 & 56 & 24 & 49 & 16 & 0 & & & 140 & 92.69 \\
\hline 6 & 0 & 67 & 52 & 27 & 45 & 4 & 75 & 0 & & & & & 137 & 41.22 \\
\hline
\end{tabular}

\section{Problem 3}

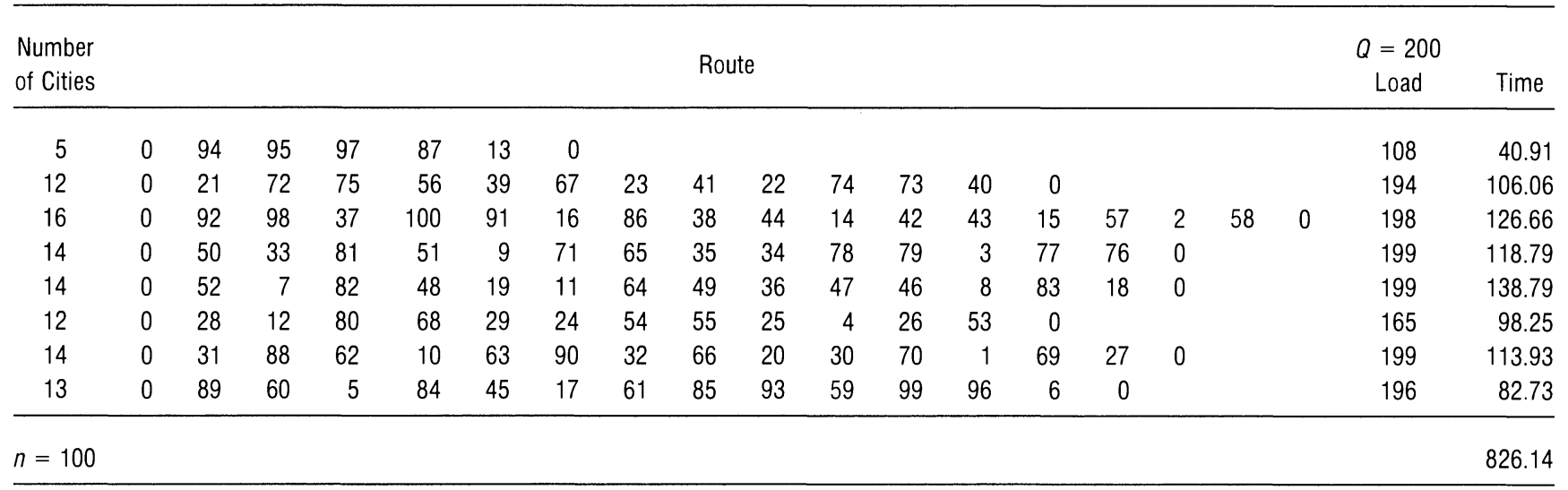

Problem 4

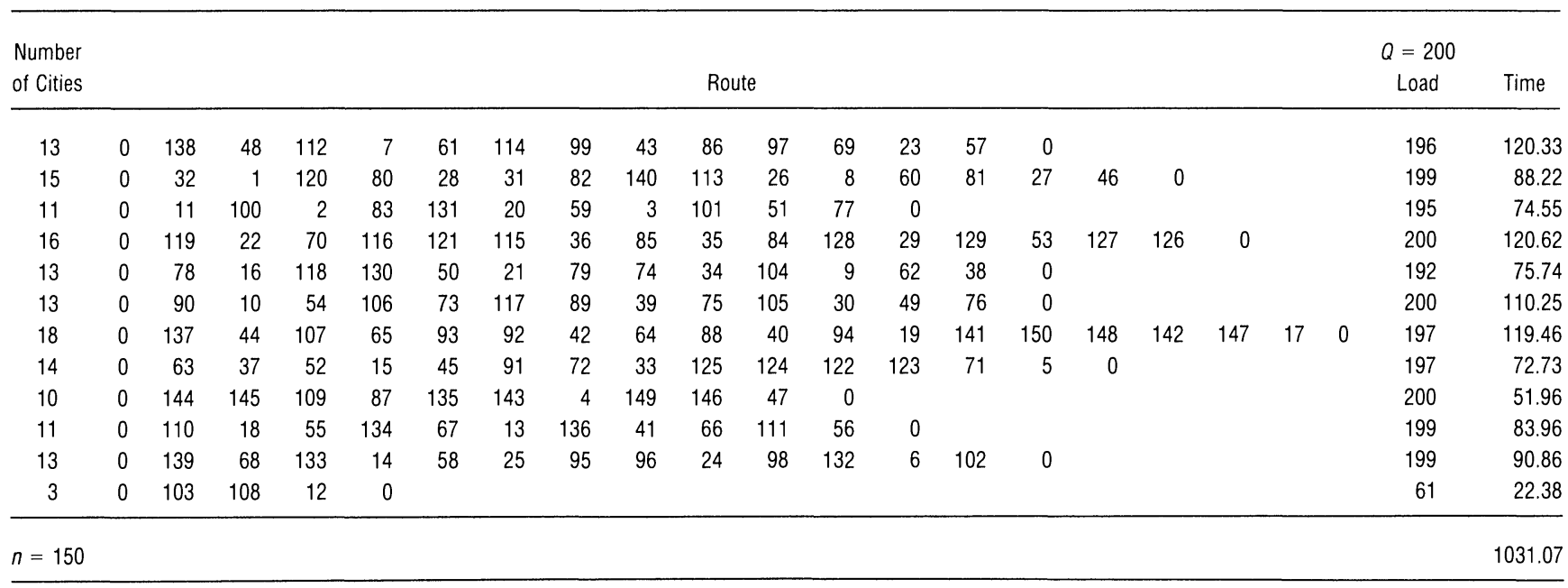


Problem 5

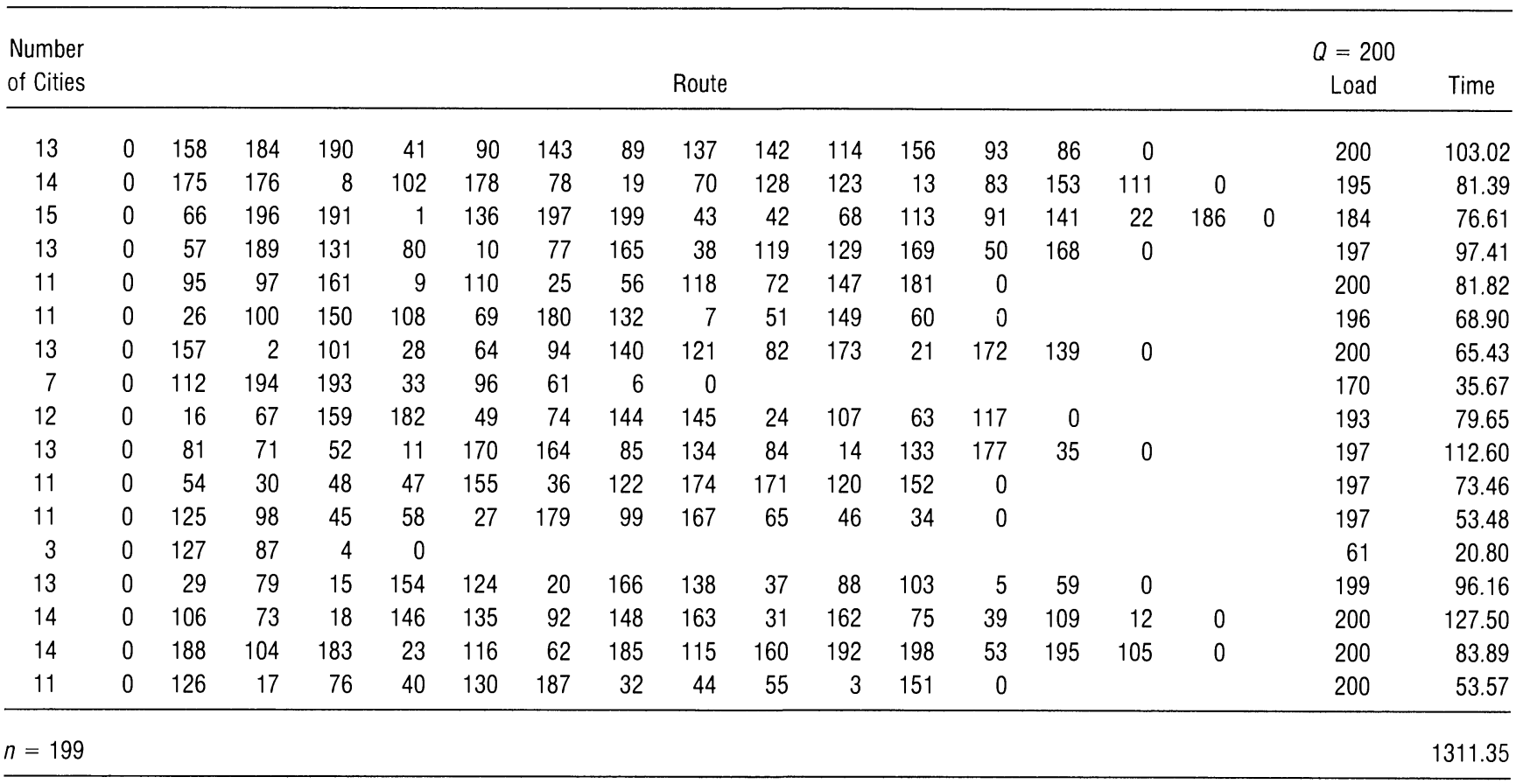

Problem 6

\begin{tabular}{|c|c|c|c|c|c|c|c|c|c|c|c|c|c|c|}
\hline 10 & 0 & 32 & 11 & 16 & 29 & 21 & 50 & 34 & 30 & 9 & 38 & 0 & 141 & 95.33 \\
\hline 10 & 0 & 12 & 37 & 44 & 15 & 45 & 33 & 39 & 10 & 49 & 5 & 0 & 155 & 99.12 \\
\hline 8 & 0 & 14 & 25 & 13 & 41 & 40 & 19 & 42 & 17 & 0 & & & 131 & 109.94 \\
\hline 4 & 0 & 18 & 4 & 47 & 46 & 0 & & & & & & & 80 & 42.33 \\
\hline$n=50$ & & & & & & & & & & & & & & 555.43 \\
\hline
\end{tabular}


Problem 7

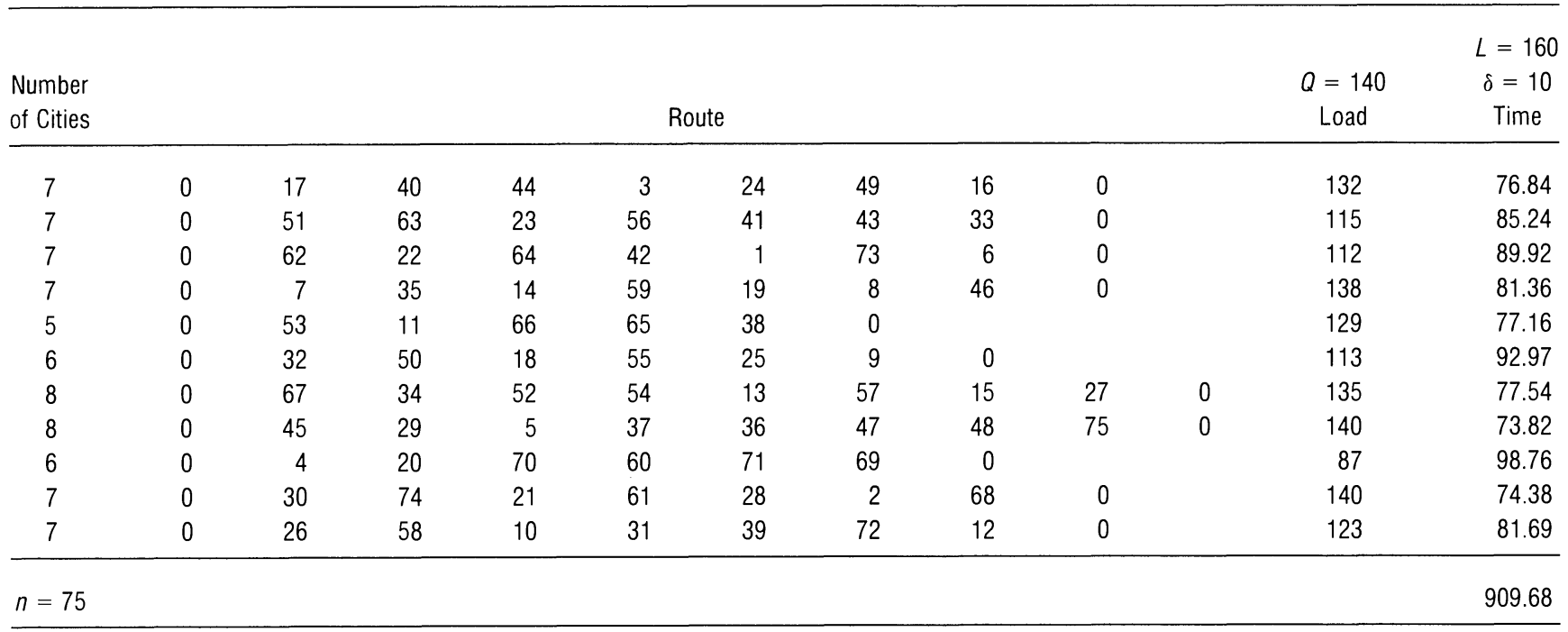

Problem 8

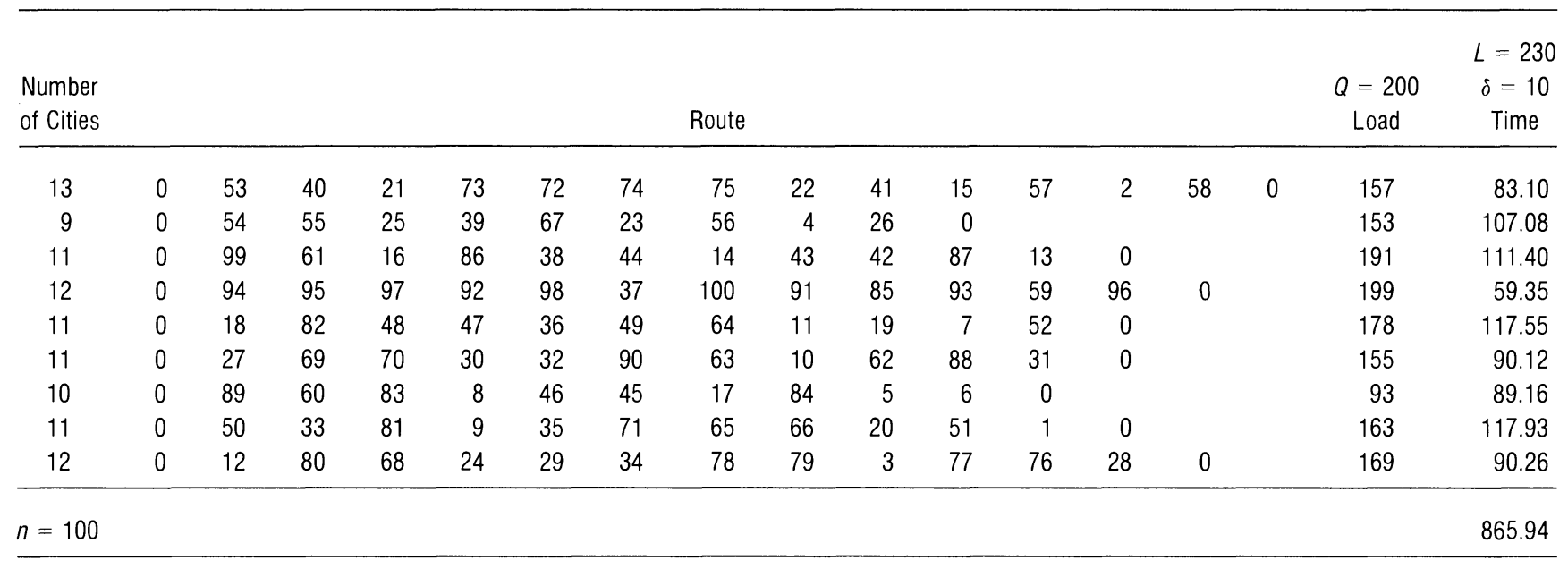


Problem 9

\begin{tabular}{|c|c|c|c|c|c|c|c|c|c|c|c|c|c|c|c|c|c|}
\hline 11 & 0 & 138 & 12 & 109 & 134 & 24 & 25 & 55 & 130 & 54 & 149 & 26 & 0 & & & 154 & 82.39 \\
\hline 10 & 0 & 5 & 84 & 17 & 113 & 86 & 140 & 38 & 14 & 100 & 95 & 0 & & & & 173 & 99.74 \\
\hline 9 & 0 & 51 & 103 & 71 & 136 & 65 & 66 & 20 & 122 & 1 & 0 & & & & & 119 & 108.57 \\
\hline 11 & 0 & 18 & 82 & 124 & 46 & 45 & 125 & 8 & 114 & 83 & 60 & 118 & 0 & & & 168 & 88.80 \\
\hline 10 & 0 & 50 & 102 & 33 & 81 & 120 & 9 & 135 & 35 & 34 & 78 & 0 & & & & 166 & 91.04 \\
\hline 12 & 0 & 53 & 40 & 21 & 73 & 74 & 133 & 22 & 41 & 145 & 115 & 2 & 58 & 0 & & 142 & 64.60 \\
\hline 10 & 0 & 105 & 110 & 4 & 139 & 39 & 67 & 23 & 56 & 75 & 72 & 0 & & & & 193 & 96.13 \\
\hline 12 & 0 & 31 & 10 & 108 & 90 & 32 & 131 & 128 & 30 & 70 & 101 & 69 & 132 & 0 & & 168 & 79.99 \\
\hline
\end{tabular}

\section{Problem 10}

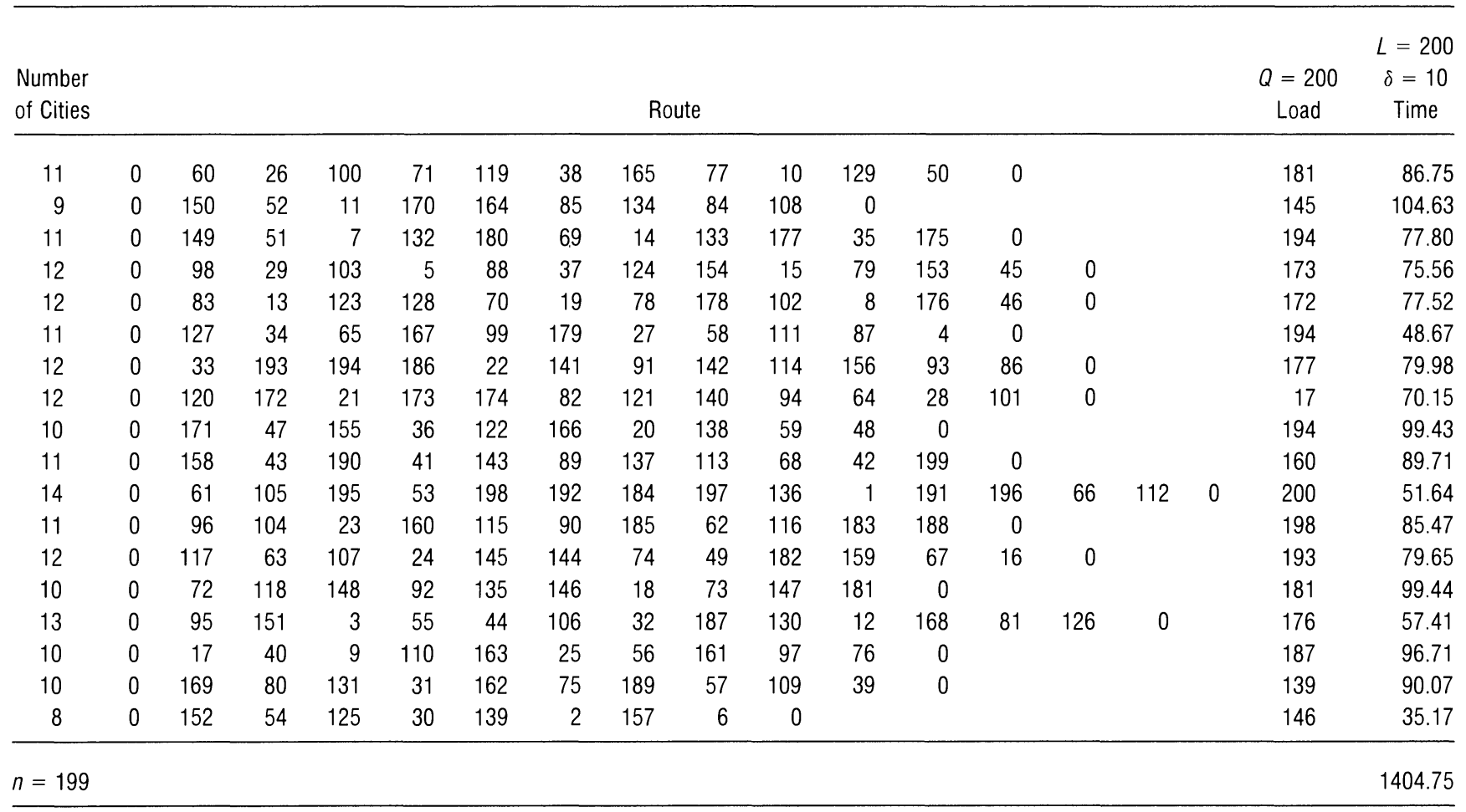


Problem 11

\begin{tabular}{|c|c|c|c|c|c|c|c|c|c|c|c|c|c|c|c|c|c|c|c|c|c|}
\hline 21 & 0 & 109 & 21 & 20 & 23 & 26 & 28 & 32 & 35 & 29 & 36 & 34 & 31 & 30 & 33 & 27 & 24 & 22 & 25 & & \\
\hline & 19 & 16 & 17 & 0 & & & & & & & & & & & & & & & & 197 & 207.94 \\
\hline 16 & 0 & 40 & 43 & 45 & 48 & 51 & 50 & 49 & 47 & 46 & 44 & 41 & 42 & 39 & 38 & 37 & 95 & 0 & & 200 & 199.63 \\
\hline 16 & 0 & 88 & 2 & 1 & 3 & 4 & 5 & 6 & 7 & 9 & 10 & 11 & 15 & 14 & 13 & 12 & 8 & 0 & & 199 & 134.96 \\
\hline 18 & 0 & 82 & 111 & 86 & 85 & 89 & 91 & 90 & 114 & 18 & 118 & 108 & 83 & 113 & 117 & 84 & 112 & 81 & 119 & & \\
\hline & 0 & & & & & & & & & & & & & & & & & & & 188 & 66.96 \\
\hline$n=120$ & & & & & & & & & & & & & & & & & & & & & 1042.11 \\
\hline
\end{tabular}

\section{Problem 12}

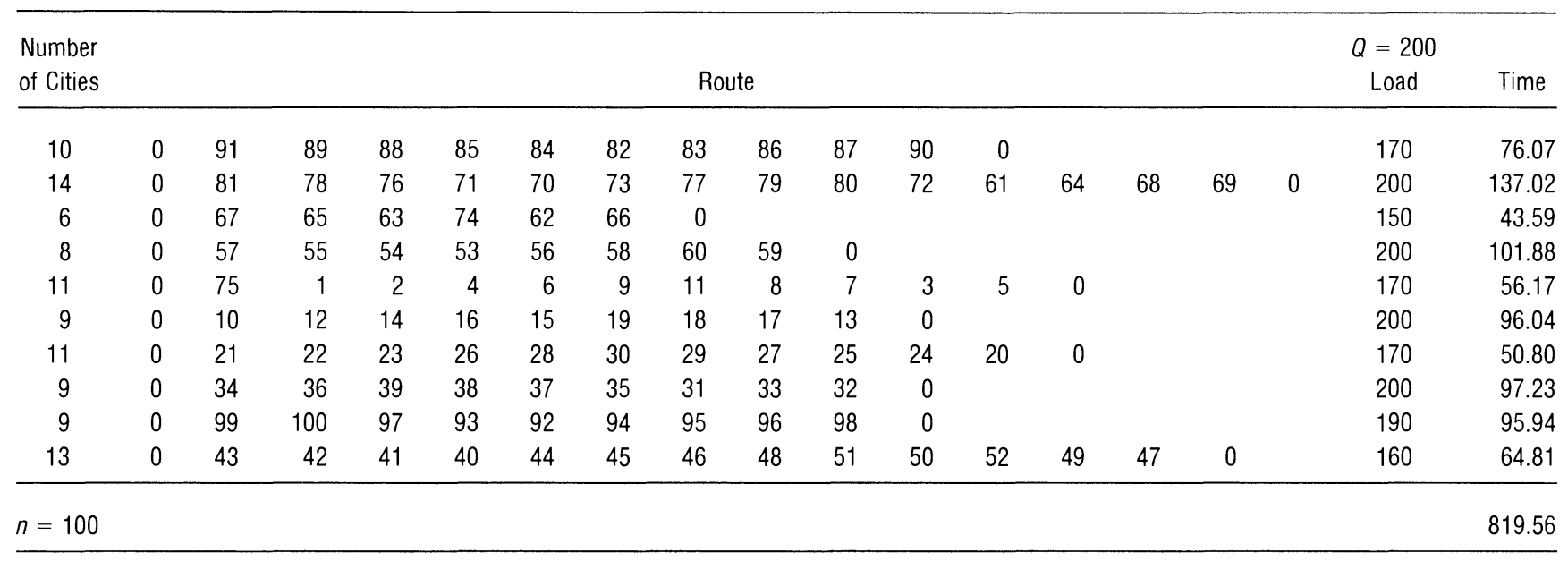

\section{Problem 13}

\begin{tabular}{|c|c|c|c|c|c|c|c|c|c|c|c|c|c|c|c|c|c|}
\hline & & & & & & & & & & & & & & & & & \\
\hline 12 & 0 & 113 & 83 & 2 & 1 & 3 & 4 & 5 & 6 & 109 & 114 & 90 & 91 & 0 & & 138 & 113.85 \\
\hline 12 & 0 & 18 & 118 & 108 & 8 & 12 & 13 & 14 & 15 & 11 & 10 & 9 & 7 & 0 & & 153 & 117.70 \\
\hline 10 & 0 & 21 & 20 & 26 & 23 & 25 & 24 & 22 & 19 & 16 & 17 & 0 & & & & 123 & 170.53 \\
\hline 10 & 0 & 40 & 43 & 45 & 48 & 51 & 65 & 61 & 57 & 54 & 52 & 0 & & & & 143 & 218.37 \\
\hline 10 & 0 & 53 & 55 & 58 & 56 & 60 & 63 & 66 & 64 & 62 & 59 & 0 & & & & 125 & 207.08 \\
\hline 11 & 0 & 73 & 71 & 74 & 72 & 75 & 78 & 80 & 79 & 77 & 76 & 68 & 0 & & & 141 & 136.49 \\
\hline 12 & 0 & 120 & 70 & 69 & 67 & 98 & 110 & 115 & 97 & 94 & 93 & 96 & 95 & 0 & & 144 & 118.66 \\
\hline 13 & 0 & 88 & 82 & 111 & 86 & 87 & 92 & 89 & 85 & 112 & 84 & 117 & 81 & 119 & 0 & 146 & 45.80 \\
\hline
\end{tabular}


Problem 14

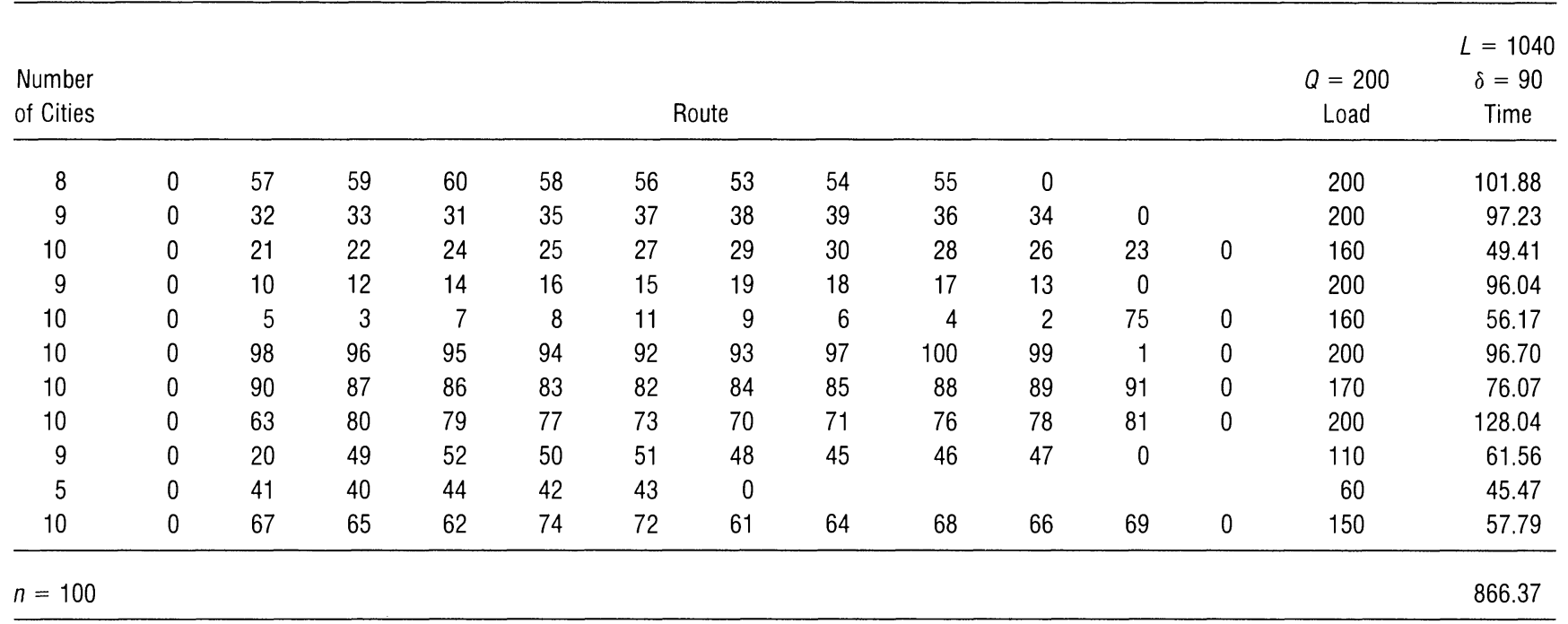

\section{References}

Altinkemer, K. and B. Gavish, "Parallel Savings Based Heuristic for the Delivery Problem," Oper. Res., 39 (1991), 456-469.

Beasley, J. E., "Route First-Cluster Second Methods for Vehicle Routing," Omega, 11 (1983), 403-408.

Bodin, L. D., B. L. Golden, A. A. Assad, and M. O. Ball, "Routing and Scheduling of Vehicles and Crews. The State of the Art," Computers \& Oper. Res., 10 (1983), 69-211.

Cheshire, I. M., A. M. Malleson, and P. F. Naccache, "A Dual Heuristic for Vehicle Scheduling," J. Operational Res. Soc ., 33 (1982), 5161.

Christofides, N., "Vehicle Routing," The Traveling Salesman Problem. A Guided Tour of Combinatorial Optimization, E. L. Lawler, J. K. Lenstra, A. H. G. Rinnooy Kan, and D. B. Shmoys (Eds.), Wiley, Chichester, 1985, 431-448.

- A. Mingozzi, and P. Toth, "The Vehicle Routing Problem," Combinatorial Optimization, N. Christofides, A. Mingozzi, P. Toth, and C. Sandi (Eds.), Wiley, Chichester, 1979, 315-338.

Clarke, G. and J. W. Wright, "Scheduling of Vehicles from a Central Depot to a Number of Delivery Points," Oper. Res., 12 (1964), 568-581.

Cornuejols, G. and F. Harche, "Polyhedral Study of the Capacitated Vehicle Routing Problem," Math. Prog., 60 (1993), 21-52.

Desrochers, M. and T. W. Verhoog, "A Matching Based Savings Algorithm for the Vehicle Routing Problem," Cahier du GERAD G-89-04. École des Hautes Études Commerciales de Montréal, 1989.

Fisher, M. L., “Optimal Solution of Vehicle Routing Problems and Minimum $k$-Trees," Report 89-12-13. Decision Sciences Department, The Wharton School, Philadelphia, PA, 1989.

— and R. Jaikumar, "A Generalized Assignment Heuristic for Vehicle Routing," Networks, 11 (1981), 109-124.
Garey, M. R. and D. S. Johnson, Computers and Intractability: A Guide to the Theory of NP-Completeness, Freeman, San Francisco, 1979.

Gendreau, M., A. Hertz, and G. Laporte, "New Insertion and PostOptimization Procedures for the Traveling Salesman Problem," Oper. Res., 40 (1992), 1086-1094.

Gillett, B. and L. Miller, "A Heuristic Algorithm for the Vehicle Dispatch Problem," Oper. Res., 22 (1974), 340-349.

Glover, F., "Heuristic for Integer Programming Using Surrogate Constraints," Decision Sciences, 8 (1977), 156-166.

—, "Tabu Search, Part I," ORSA J. Computing, 1 (1989), 190-206.

_ , "Tabu Search, Part II," ORSA J. Computing, 2 (1990), 4-32.

— and M. Laguna, "Tabu Search," Modern Heuristic Techniques for Combinatorial Problems, C. Reeves (Ed.), Blackwell Scientific Publications, Oxford, 1993, 70-150.

- E. Taillard, and D. de Werra, "A User's Guide to Tabu Search," Annals of Operations Research, 41 (1993), 3-28.

Golden, B. L. and A. A. Assad, "Vehicle Routing: Methods and Studies," North-Holland, Amsterdam, 1988.

Hadjiconstantinou, E. and N. Christofides, "An Optimal Procedure for Solving Basic Vehicle Routing Problems," presented at the 35th Annual Conference of the Canadian Operational Research Society, Halifax, Canada, 1993.

Haimovich, M. and A. H. G. Rinnooy Kan, "Bounds and Heuristics for Capacitated Routing Problems," Math. Oper. Res., 10 (1985), 527-542.

Harche, F. and P. Raghavan, "A Generalized Exchange Heuristic for the Capacitated Vehicle Problem," Working Paper, Stern School of Business, New York University, 1991.

Hertz, A., "Finding a Feasible Course Schedule Using Tabu Search," Discrete Applied Math., 35 (1992), 255-270.

Kirkpatrick, S., Gelatt, C. D. Jr., and M. P. Vecchi, "Optimization by Simulated Annealing," Science, 220 (1983), 671-680. 
Laporte, G., "The Vehicle Routing Problem: An Overview of Exact and Approximate Algorithms," European J. Operational Res., 59 (1992), 345-358.

- and Y. Nobert, "Exact Algorithms for the Vehicle Routing Problem," Surveys in Combinatorial Optimization, S. Martello, G. Laporte, M. Minoux and C. Ribeiro (Eds.), North-Holland, Amsterdam, 1987, 147-184.

Metropolis, N., A. W. Rosenbluth, M. N. Rosenbluth, A. H. Teller, and E. Teller, "Equation of State Calculations by Fast Computing Machines," J. Chemical Physics, 21 (1953), 1087-1091.

$\rightarrow$ Mole, R. H., "The Curse of Unintended Rounding Error: A Case from the Vehicle Scheduling Literature," J. Operational Res. Society, 34 (1983), 607-613.

$-\rightarrow$ and S. R. Jameson, "A Sequential Route-Building Algorithm Employing a Generalised Savings Criterion," Operational Res. Quarterly, 27 (1976), 503-511.

Noon, C. E., J. Mittenthal, and R. Pillai, "A TSSP+1 Decomposition Approach for the Capacity-Constrained Vehicle Routing Problem," Working Paper, Management Science Program, The University of Tennessee, Knoxville, TN, 1991.

Or, I., "Traveling Salesman-Type Combinatorial Optimization Problems and Their Relation to the Logistics of Regional Blood Banking, " Ph.D. Dissertation, Northwestern University, Evanston, IL, 1976.

Osman, I. H., "Metastrategy Simulated Annealing and Tabu Search Algorithms for Combinatorial Optimization Problems," Ph.D. Dissertation, The Management School, Imperial College, London, 1991.
_- "Metastrategy Simulated Annealing and Tabu Search Algorithms for the Vehicle Routing Problem," Annals of Oper. Res., 41 (1993), 421-451.

Pureza, V. M. and P. M. França, “Vehicle Routing Problems via Tabu Search Metaheuristic," Publication CRT-747, Centre de recherche sur les transports, Montréal, 1991.

Riordan, J., An Introduction to Combinatorial Analysis, Wiley, New York, 1958.

Semet, F. and E. Taillard, "Solving Real-Life Vehicle Routing Problems Efficiently Using Taboo Search," Annals of Oper. Res., 41 (1993), 469-488.

Stewart, W. R. Jr. and B. L. Golden, "A Lagrangian Relaxation Heuristic for Vehicle Routing," European J. Operational Res., 15 (1984), $84-88$.

Taillard, E., "Robust Taboo Search for the Quadratic Assignment Problem," Parallel Computing, 17 (1991), 433-445.

- , "Parallel Iterative Search Methods for Vehicle Routing Problems." Working Paper ORWP 92/03, Département de Mathématiques, École Polytechnique Fédérale de Lausanne, Switzerland, 1992.

Toth, P., "Heuristic Algorithms for the Vehicle Routing Problem," presented at the Workshop on Routing Problems, Hamburg, 1984.

Willard, J. A. G., "Vehicle Routing Using $r$-Optimal Tabu Search," M.Sc. Dissertation. The Management School, Imperial College, London, 1989.

Accepted by Awi Federgruen; received July 15, 1991. This paper has been with the authors $7 \frac{1}{2}$ months for 2 revisions. 